\title{
Improving Analytical Efficiency of EDS using a Newly-designed X-ray Detecting System for Aberration Corrected 300 kV Microscope
}

Takeo Sasaki, Hidetaka Sawada, Eiji Okunishi, Yu Jimbo, Yorinobu Iwasawa, Koji Miyatake, Shuichi Yuasa, and Toshikatsu Kaneyama

JEOL Ltd., EM Business Unit, 3-1-2 Musashino, Akishima, Tokyo 196-8558, Japan

Performance of elemental analysis with energy-dispersive X-ray spectroscopy (EDS) has been improved by using large-sized silicon drift detectors (SDDs) [1]. The detectors are required to place closer to the specimen holder to obtain more counts of X-ray signals because the solid angle of the X-ray detectors increases. The positions, sizes and number of the detectors have not been satisfactorily optimized to attain a high analytical efficiency, since the space to place the detectors is limited. We have developed a new X-ray detecting system for JEM-ARM300F that equips a cold field emission electron source. The key point of this system is to renovate an objective lens pole-piece and a specimen holder in addition to the SDDs.

A newly designed wide gap pole-piece (WGP) accommodates new SDDs to place closer to the specially designed analytical holder so that a larger amount of X-ray signal can be detected with a very high efficiency. Figure 1 compares peak intensities of $\mathrm{Ni}-\mathrm{K}$ obtained from a $\mathrm{NiOx}$ thin film at $80 \mathrm{kV}$ with SDD1, SDD2, and SDD1 + SDD2 (Dual). The intensity of the SDD2 is approximately 1.8 times larger than that of SDD1 due to the shorter distance between the detector (SDD2) and a specimen. The SDD2 is placed in the direction of the sample holder rod and is located at a closer position to the specimen than SDD1, because the special specimen holder has a shorter length in the holder rod direction. Table 1 shows the solid angles and take off angles of the SDD1 and SDD2. The solid angle of SDD2 (1.08 sr) is about two times larger than that of SDD1 (0.55 sr), which accords well with the intensity ratio of the Ni$\mathrm{K}$ spectra. Figure 2 shows atomic resolution EDS maps of $\mathrm{SrTiO}_{3}[100]$ taken at $80 \mathrm{kV}$. Sr and Ti columns are clearly seen. It is worth to note that Ti-O columns and $\mathrm{O}$ columns are visible at an acquisition time of 13.5 minutes (number of pixels $=128 \times 128$ ). This proves a high analytical efficiency of the present system. It is clear from the short acquisition time that this X-ray detecting system is a very powerful tool for elemental analysis of damage-susceptible materials such as carbon materials.

A spherical aberration coefficient (Cs) and a chromatic aberration coefficient (Cc) of WGP pole-piece $(\mathrm{Cs}=1.6 \mathrm{~mm}, \mathrm{Cc}=2.4 \mathrm{~mm})$ are larger than those of $\mathrm{FHP}$ pole-piece $(\mathrm{Cs}=0.7 \mathrm{~mm}, \mathrm{Cc}=1.3 \mathrm{~mm})$. (FHP is an optional objective lens pole-piece for ultra-high resolution in JEM-ARM300F and enables to resolve the Si to Si dumbbell of $45 \mathrm{pm}$ in $\mathrm{Si}[114]$ sample [2].) We have tested the imaging performance of WGP. Figure 3(a) shows a Cs-corrected high angle annular dark field scanning transmission electron microscopy (HAADF-STEM) image (averaged with four sequential raw images) of Si[112] taken at 300 $\mathrm{kV}$ with a dwell time of $38 \mu \mathrm{sec} / \mathrm{pix}$. The Si to Si dumbbell of $78 \mathrm{pm}$ is clearly resolved. The Fourier transform of the image exhibits spots of $(73 \mathrm{pm})^{-1}$ as well as $(78 \mathrm{pm})^{-1}$ as shown in Figure 3(b). Figure 3(c) shows Cs-corrected TEM image of $\beta-\mathrm{Si}_{3} \mathrm{~N}_{4}[0001]$. The Si to $\mathrm{N}$ dumbbell of $95 \mathrm{pm}$ is clearly imaged. These results have revealed ultra-high resolution STEM and TEM imagings are possible even with the WGP pole-piece. The wide gap permits a higher specimen tilting angle, and gives enough room for special holders of heating, cooling, gas environment etc. We emphasize that the WGP can be used for observations of many purposes. 
References:

[1] S Kawai et al, Microsc. Microanal. 20. Suppl. 3 (2014) p.1150-1151.

[2] H Sawada et al, Microsc. Microanal. 20. Suppl. 3 (2014) p.124-125.

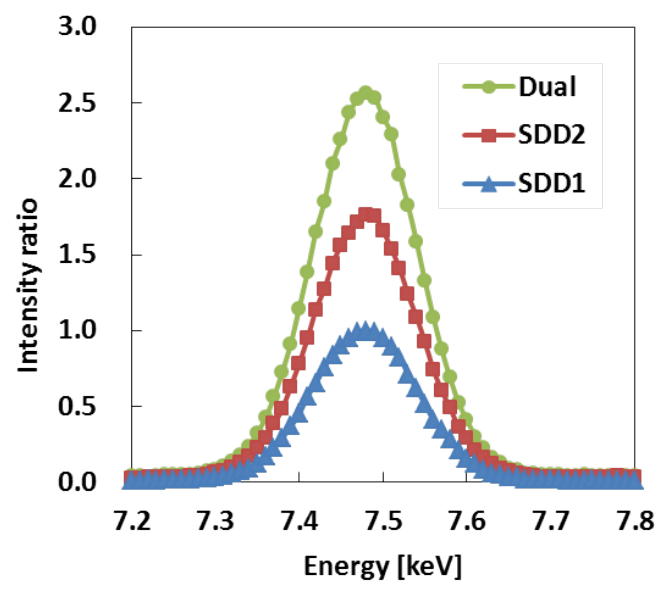

\begin{tabular}{|c|c|c|}
\hline & \multicolumn{2}{|c|}{$\begin{array}{c}\text { WGP } \\
\text { (Dual-SDD) }\end{array}$} \\
\hline & SDD1 & SDD2 \\
\hline Solid angle [sr] & 0.55 & 1.08 \\
\hline Take-off angle [deg] & 25 & 29 \\
\hline
\end{tabular}

Table 1 Solid angles and take off angles of SDD1 and SDD2 with the use of WGP.

Figure 1. EDS spectra taken from a NiOx thin film at $80 \mathrm{kV}$. Blue triangles, red squares, and light green circles correspond to SDD1, SDD2, and SDD1+SDD2 (Dual), respectively. The intensities of the spectra were normalized using the peak intensity of Ni-K obtained with the SDD1.

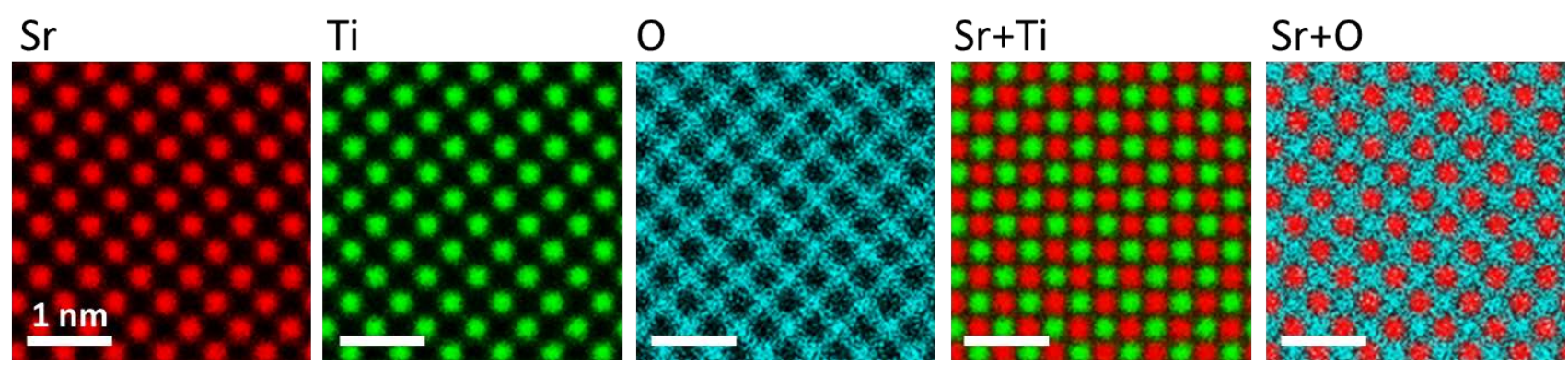

Figure 2. EDS elemental maps (radial-difference-filtered) of $\mathrm{SrTiO}_{3}[001]$ taken at $80 \mathrm{kV}$ with a probe current of $150 \mathrm{pA}$, a convergence semi-angle of $24 \mathrm{mrad}$, a map size of $128 \times 128$ pixels and an acquisition time of 13.5 minutes. Each scale bar represents $1 \mathrm{~nm}$.
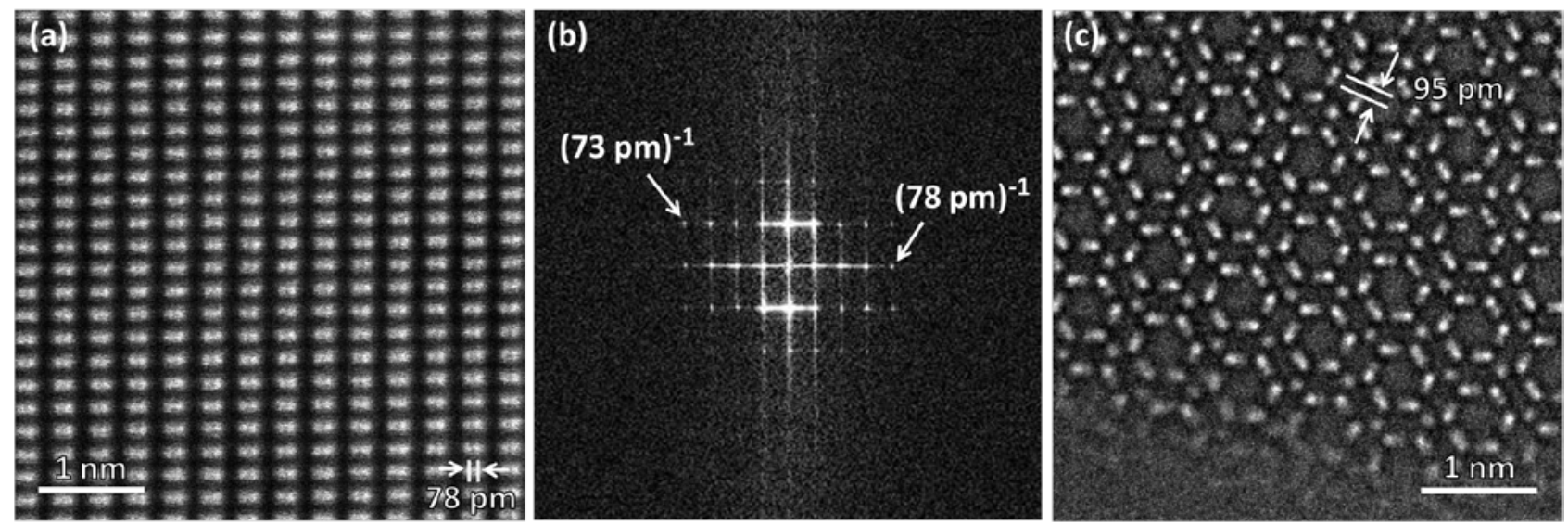

Figure 3. (a) Cs-corrected HAADF-STEM image of Si[112] taken at $300 \mathrm{kV}$ (WGP pole-piece) with a dwell time of $38 \mu \mathrm{sec} /$ pix $(512 \times 512$ pixels), averaged with four sequential raw images. (b) Fourier transform (in power) of the image shown in (a). (c) Cs-corrected TEM image (raw data) of $\beta$-Si ${ }_{3} \mathrm{~N}_{4}[0001]$ taken at $300 \mathrm{kV}$ (WGP pole-piece). 